\section{Band 26, Heft 2, März 1999}

G egründet 1973 als «D ie Infusionstherapie» durch H. Reissigl, H. G robecker, U. H enneberg, K.H . Bäßler

B isherige $\mathrm{H}$ erausgeber und Schriftleiter:

H .-D . Viering (1973-1977), A . G rünert (1982-1992),

G. K leinberger (1982-1988), K. Widhalm (1988-1992),

G. Wolfram (1990-1992), J. E ckart (1992-1997),

H. Forst (1992-1997), K. M eßmer (1992-1997),

K. Peter (1992-1997), K. H. U sadel (1992-1997),

B. Z wißler (1995-1997)
Offizielles Organ der Deutschen Gesellschaft für Transfusionsmedizin und Immunhämatologie

\section{Verantwortlicher Herausgeber}

V. K retschmer, M arburg

\section{Herausgeber}

W. D zik, B oston, M A

H.G. K lein, B ethesda, M D

W. Sibrowski, M ünster

W. Stangel, $\mathrm{H}$ annover

\section{Schriftleitung}

K. G utensohn, H amburg

A. Sputtek, $\mathrm{H}$ amburg

\title{
Ressort-Herausgeber
}

\section{Blutprodukte}

G. H olzberger, K assel

H. K lüter, Lübeck

$K$ linische Transfusionsmedizin

M.U. H eim, M agdeburg

A.G. Salama, B erlin

Transplantation

G. B ein, Gießen

M. W iesneth, U Im

Immunhämatologie

V. K iefel, Leipzig

H.H. Sonneborn, D reieich

$\mathrm{H}$ ämostaseologie

M. K öhler, Göttingen

E. Seifried, Frankfurt/M .
Sicherheit, Risiken und

$\mathrm{N}$ ebenwirkungen

G. Caspari, G ießen

U.T. Seyfert, H omburg/Saar

R echtsfragen, Standards

R. Dörner, Köln

N. Petersen, D ortmund

Weiterbildung

M. B öck, M agdeburg

A. Greinacher, G reifswald

A utologe $\mathrm{H}$ ämotherapie, blutsparende Verfahren

J. Biscoping, K arlsruhe

W. M empel, M ünchen

W. Schleinzer, H eilbronn

\section{Wissenschaftlicher Beirat}

\section{H. B ardenheuer, $\mathrm{H}$ eidelberg}

J.-F. Baron, Paris

B. Blauhut, L inz

H. B orberg, Köln

U.B. B rückner, U Im

H.W. D oerr, Frankfurt/M.

R. E ckstein, E rlangen

H. Forst, A ugsburg

$L$. Frey, M ünchen

W. Gerlich, G ießen

E. G ötz, D armstadt

H. G ombotz, G raz

L. G ürtler, M ünchen

P. H anfland, B onn
E. H ansen, R egensburg
P. Höcker, Wien
J.P. Isbister, Sydney
H. K iesewetter, B erlin
H.-J. Kolb, M ünchen
B. Kubanek, U Im
P. K ühnl, $\mathrm{H}$ amburg
H. L aubenthal, B ochum
K. M eßmer, M ünchen
N. M üller, E ssen
G. M üller-B erghaus, B ad N auheim
E . N orthoff, Tübingen
U.E. N ydegger, Bern
$K$. Peter, $M$ ünchen
R. Pietersz, A msterdam
G.F. R iedler, L uzern
D. R oelcke, $H$ eidelberg
G. Schmitz, R egensburg
N. Schmitz, K iel
D. Schönitzer, Innsbruck
W. Schramm, M ünchen
D. Spahn, Zürich
L. Sunder-Plassmann, U Im
D. Voak, Cambridge, U K
E. Wenzel, H omburg/Saar
K. Werdan, $\mathrm{H}$ alle/Saale
R. Zander, M ainz
B. Z wißler, M ünchen 
B ibliographische D ienste:

Biological A bstracts

C urrent Contents/C linical M edicine

Excerpta M edica/E M BA SE

$M$ edical D ocumentation Service

$R$ eference $U$ pdate

$R$ esearch A lert

Science Citation Index

SCISE A RCH D atabase

S. K arger · M edical and Scientific Publishers · B asel · Freiburg · Paris · London · N ew Y ork · N ew D elhi · B angkok · Singapore · Tokyo $\cdot$ Sydney

Die Zeitschrift erscheint zweimonatlich; pro Jahr erscheint 1 Band zu je 6 Heften. DGTI-Mitglieder erhalten die Zeitschrift im $\mathrm{R}$ ahmen ihrer M itgliedschaft. B ezugspreis für J ahrgang 26, 1999, DEM 198,- / CH F 154,-, einschließlich M W St. zuzüglich Postgebühren. Der A bonnementpreis ist im voraus zahlbar. D as A bonnement der Z eitschrift läuft weiter, wenn es nicht spätestens 4 W ochen vor A bschluß eines Bandes abbe stellt wird. A bonnementbestellungen können bei jeder Buchhandlung oder direkt beim $V$ erlag aufgegeben werden:

\section{Bundesrepublik}

Deutschland:

$\mathrm{S}$. K arger $\mathrm{G} \mathrm{mbH}$

Lörracher Str. 16a,

D-79115 Freiburg,

Telefon +49 761452070 ,

Telefax +497614520714,

Telefax +497614520714,
E-mail kargergmbh@aol.com
Postbank M ünchen

Postbank Mur unch
$6140-808$.

Ü brige L änder:
S. K arger A G,
A llschwilerstr. 10
Postfach
CH-4009 B asel,
Telefon +41 6130611 11,
Telefax +41 613061234,
E-mail K arger@K arger.ch

S. K arger A G,

A llschwilerstr. 10

Postfach

Telefon +41613061111

E-mail K arger@K arger.ch
A nzeigen: S. K arger

Verlag für Medizin und Naturwissenschaften $\mathrm{GmbH}$, Lörracher Str. 16a, D-79115 Freiburg,

Telefon +49761452070

G ültig ist die Preisliste N r. 14 vom 1. J anuar 1999.

Für den Inhalt außerhalb des redaktionellen Teiles (insbesondere A nzeigen, Industrieinformationen, Pressezitate und K ongreßinformationen) übernehmen Schriftleitung, B eirat und Verlag keine $\mathrm{G}$ ewähr.

Eine Markenbezeichnung kann warenzeichenrechtlich geschützt sein, auch wenn bei ihrer Verwendung in dieser Z eitschrift das $\mathrm{Z}$ eichen ${ }^{\circledR}$ oder ein anderer $\mathrm{H}$ inweis auf etwa bestehende Schutzrechte fehlen sollte. Für Satzfehler, insbesondere bei $D$ osierungsangaben, wird keine $\mathrm{G}$ ewähr übernommen

D ie Z eitschrift sowie alle in ihr enthaltenen einzelnen B eiträge Die Z eitschrift sowie alle in ihr enthaltenen einzelnen Beitrage und A bbildungen sind urheberrechllich geschützt. J ede Verwertung, die nicht ausdrücklich vom $U$ rheberrechtsgesetz zugelassen ist, bedarf der vorherigen Z ustimmung des V erlags. D as gilt insbesondere für V ervielfältigungen, B earbeitungen, Ü bersetzungen, Mikroverfilmungen und die Einspeicherung und Verarbeitung in elektronischen Systemen. (c) Copyright 1999 by S. K arger

Verlag für M edizin und $\mathrm{N}$ aturwissenschaften $\mathrm{G}$ mbH Lörracher Str. 16a, D-79115 Freiburg

Verlagsleitung und presserechtlich verantwortlich: Sibylle $\mathrm{H}$ opf Produktionsleitung: G eorg B runner A nzeigenleitung: Susanne M eister

H erstellung: K onkordia D ruck $\mathrm{GmbH}$ Eisenbahnstraße 31, D-77815 Büh

\section{KARGER}


Ü bersichtsarbeiten

78 A spekte der $\mathbf{Q}$ ualitätssicherung im $\mathbf{R}$ ahmen der peripheren B lutstammzelltransplantation Cassens, U.; A hlke, C.; Sibrowski, W. (M ünster)

85 Z ytokin-induzierte M obilisation peripherer B lutstammzellen aus dem Steady-state bei Patienten mit soliden Tumoren und hämatologischen Neoplasien K röger, N.; Z eller, W.; H assan, H .T.; K rüger, W.; R enges, H .; R üssmann, B.; Fuchs, N.; H ummel, K.; R auhöft, C.; G utensohn, K .; Löliger, C.; Erttmann, R.; Kühnl, P.; Zander, A .R . (H amburg)

92 Stammzellmobilisation bei gesunden Spendern: Derzeitiger Stand D reger, P.; Schmitz, H . (Kiel)

96 Nachweis disseminierter epithelialer Tumorzellen: Methoden und klinische $A$ nwendung Pantel, K .; Witter, K. (M ünchen)

Originalarbeiten

103 B edeutung der Vorläuferzellwerte des peripheren B lutes der $\mathbf{R}$ uhehämatopoese für die A bschätzung der A nzahl an B lutvorläuferzellen, die nach einer $\mathbf{G}$ ranulozytenkoloniestimulierender-Faktor-unterstützten Chemotherapie mobilisiert werden

Fruehauf, S.; Schmitt, K.; Veldwijk, M .R .; Schiedlmeier, B.; M artin, S.; Zeller, W..;; Haas, R. (H eidelberg)

110 D as Mannheimer Nabelschnurblut-Projekt: E rfahrungen zur Sammlung und A ufarbeitung der ersten $\mathbf{8 8 0}$ unverwandten Transplantate aus P lazentablut E ichler, H.; R ichter, E .; Leveringhaus, A .; Zieger, W.; Watz, E .; Friedmann, G.; K erowgan, M .; Goldmann, S.F. (M annheim)

115 Phänotyp und F unktion antigenpräsentierender dendritischer $Z$ ellen, die aus M onozyten des peripheren $B$ luts generiert wurden B auer, Y.; äger, C.; K ramer, M .D .; Wallich, R . (H eidelberg)
R eview A rticles

78 A spects of Q uality A ssurance in the Preparation of Peripheral B lood Stem C ells for Transplantation Cassens, U.; A hlke, C.; Sibrowski, W. (M ünster)

85 Steady-State M obilization of Peripheral B lood Progenitor Cells by Cytokines A lone in Patients with Solid Tumors or Hematological Malignancies K röger, N.; Z eller, W.; H assan, H .T.; K rüger, W.; R enges, H .; R üssmann, B.; Fuchs, N.; H ummel, K.; R auhöft, C.; G utensohn, K .; Löliger, C.; E rttmann, R.; Kühnl, P.; Zander, A .R . (H amburg)

92 Stem Cell M obilization in H ealthy D onors: C urrent Status

D reger, P.; Schmitz, H . (K iel)

96 D etection of D isseminated E pithelial Tumor C ells: Methods and C linical Implications Pantel, K .; Witter, K. (M ünchen)

\section{Original A rticles}

103 R elevance of Peripheral B lood Progenitor Cell C ounts during Steady-State $\mathrm{H}$ ematopoiesis to $\mathrm{E}$ stimate the N umber of Progenitor Cells M obilized following G ranulocyte C olony-Stimulating Factor Supported Chemotherapy

Fruehauf, S.; Schmitt, K.; Veldwijk, M .R .; Schiedlmeier, B.; M artin, S.; Zeller, W.J.; H aas, R . (H eidelberg)

110 The M annheim Cord B lood Project: Experience in Collection and Processing of the First 880 B anked U nrelated C ord B lood Transplants

Eichler, H .; R ichter, E .; L everinghaus, A .; Zieger, W.; Watz, E .; Friedmann, G.; K erowgan, M .; Goldmann, S.F. (M annheim)

115 Phenotype and $F$ unction of $A$ ntigen-Presenting $D$ endritic C ells G enerated from Peripheral B lood M onocytes B auer, Y.; äger, C.; K ramer, M .D.; Wallich, R . (H eidelberg) 
Infusionstherapie

Transfusionsmedizin

Infusion Therapy

Transfusion Medicine

Band 26, Heft 2, März 1999

119 B riefe an die H erausgeber

122 Tagungen und Kongresse

2 Impressum

$123 \mathrm{H}$ inweise für Autoren
Inhalt $\cdot$ Contents
Vol. 26, Issue 2, March 1999

119 L etters to the E ditors

122 M eetings and Conferences

2 Imprint

U 3 G uidelines for A uthors 